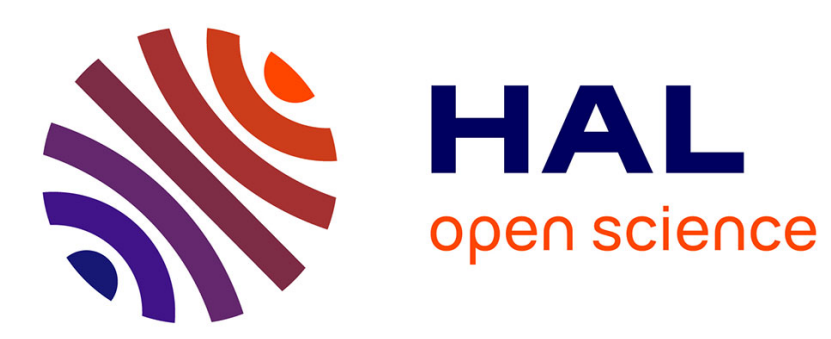

\title{
An adaptive algorithm for cohesive zone model and arbitrary crack propagation
}

Vincent Chiaruttini, Dominique Geoffroy, Vincent Riolo, Marc Bonnet

\section{To cite this version:}

Vincent Chiaruttini, Dominique Geoffroy, Vincent Riolo, Marc Bonnet. An adaptive algorithm for cohesive zone model and arbitrary crack propagation. Revue Européenne de Mécanique Numérique/European Journal of Computational Mechanics, 2012, 21, pp.208-218. 10.1080/17797179.2012.744544 . hal-00752797

\section{HAL Id: hal-00752797 \\ https://hal.science/hal-00752797}

Submitted on 16 Nov 2012

HAL is a multi-disciplinary open access archive for the deposit and dissemination of scientific research documents, whether they are published or not. The documents may come from teaching and research institutions in France or abroad, or from public or private research centers.
L'archive ouverte pluridisciplinaire HAL, est destinée au dépôt et à la diffusion de documents scientifiques de niveau recherche, publiés ou non, émanant des établissements d'enseignement et de recherche français ou étrangers, des laboratoires publics ou privés. 


\title{
An Adaptive Algorithm for Cohesive Zone Model and Arbitrary Crack Propagation
}

\author{
Vincent Chiaruttini $^{a}$, Dominique Geoffroy ${ }^{a}$, Vincent Riolo $^{a, b}$, Marc Bonnet $^{c}$ \\ ${ }^{a}$ Onera - the French Aerospace Lab, Chatillon, France \\ \{vincent.chiaruttini,dominique.geoffroy,vincent.riolo\}@onera.fr \\ ${ }^{b}$ Laboratoire de Mécanique des Solides (UMR 7649 CNRS), Ecole Polytechnique, Palaiseau, France \\ ${ }^{c}$ POems (UMR 7231 CNRS-ENSTA-INRIA), ENSTA, Paris, France (mbonnet@ensta.fr)
}

\begin{abstract}
This paper presents an approach to the numerical simulation of crack propagation with cohesive models for the case of structures subjected to mixed mode loadings. The evolution of the crack path is followed by using an adaptive method: with the help of a macroscopic branching criterion based on the calculation of an energetic integral, the evolving crack path is remeshed as the crack evolves in the simulation. Special attention is paid to the unknown fields transfer approach that is crucial for the success of the computational treatment. This approach has been implemented in the finite element code Z-Set (jointly developed by Onera and Ecole des Mines) and is tested on two examples, one featuring a straight crack path and the other involving a complex crack propagation under critical monotonous loading monotonous.
\end{abstract}

Keywords: cohesive zone model, mixed mode crack propagation, remeshing, field transfer

\section{Introduction}

The accurate prediction of crack propagation becomes increasingly necessary for a wide range of industrial applications (e.g. aerospace or automotive industries, civil engineering). Due to increasing complexity and advanced optimization methodologies, manufacturing requires ever more sophisticated design techniques and precise damage tolerance analysis for correct lifetime assessment. Critical parts, such as rotors in aircraft engines, are actively investigated for cracks, using non-destructive means of detection. Such parts, if cracked, are usually replaced immediately for safety reasons. However, crack detection technologies have limitations, and some very small initiated cracks might remain undetected during inspections. Thus, it is necessary to determine when the next inspection should occur without compromising safety. Modeling how such small cracks propagate due to fatigue loading is believed to help addressing this concern. 
During the last decade, many approaches have been developed to efficiently perform complex 3D crack growth simulations under fatigue loading (mostly based on either XFEM/Levelset methods $[3,5,15,17]$ or efficient remeshing techniques $[4,7,8]$ ), but these are mostly limited to the linear elastic fracture mechanics (LEFM) framework. Due to many technical issues, it is quite difficult to close the gap between complex LEFM and the study of cracks arising in elastic-plastic materials.

An usual alternative to LEFM is the use of cohesive zone models [2,9], which allow to model the energy dissipated in a crack opening process as a damaged interface situated on the crack path. Such approaches give the opportunity to separate the energy loss arising on the crack surface from any kind of dissipative volume material behaviour (e.g. viscoplasticity or damage). Usually, in structural mechanics, the numerical implementation of cohesive zone models is carried out using standard finite element techniques, where specific dissipative interface elements are inserted on a predefined crack path along which the crack is assumed to progress when a load is applied. Efficient tackling of mixed mode loading, where the crack path is complex and hardly predictable, is still non-trivial. A possible solution consists in inserting cohesive elements on each interface between bulk finite elements [12], but such approach greatly increases the number of degrees of freedom (DOFs), makes the predicted crack path mesh-dependent and, furthermore, adds difficulties when calibrating the model due to numerous branchings and unpredictable energy dissipation. Recently, an alternative numerical technique has been developed [10] in order to predict the crack path evolution; an averaged principal stress direction criterion is applied and a morphing approach is used to update the crack location in the mesh.

The work presented here can be seen as an extension of the latter approach, using the efficiency of remeshing techniques recently developed at Onera. When using cohesive zone models, a fine mesh is usually required near the "process zone" where energy dissipation occurs. Thus, for long crack propagation simulations, refining to mesh on the whole predefined crack path can be extremely expensive in terms of computational time (especially for 3D problems).

The article is organized as follows. Section 2 introduces the modelling strategy used for the problem at hand, and in particular the modified Crisfield cohesive zone model chosen $[1,14]$. In Section 3, an approach aiming at performing efficient cohesive zone modeling with adaptive remeshing and a predefined crack is presented, detailing the fields transfer method which is a critical part of the process. The required branching criterion and the remeshing process for crack path adaptation in mixed mode loading are explained in Section 4. Finally, Section 5 reports preliminary numerical assessments of the implementation of the proposed algorithm on the simulation of a critical crack propagation, highlighting the computational savings allowed by the proposed treatment.

\section{A continuous mechanical model with cohesive zone}

Consider a mechanical problem for a structure $\Omega$, where an initial crack is defined by a surface $\Gamma_{0}$, a prescribed displacement evolution $\boldsymbol{u}_{d}(t)$ is imposed on a subset $\partial_{u} \Omega$ of the boundary $\partial \Omega$ of the domain, while prescribed tractions $\boldsymbol{F}_{d}(t)$ are applied on the complementary portion $\partial_{F} \Omega$ of the external boundary. The structure evolution is modelled 
within the small-deformation framework, as only the crack surface geometry $\Gamma_{t}$ evolves inside the domain during the considered time interval $\left[0, t_{f}\right]$, and quasi-static conditions (e.g. slow crack propagation) are assumed. For the sake of simplicity, the bulk material is assumed to have linearly elastic constitutive properties. Besides, a cohesive zone model is used to model the energy dissipated as the crack propagates. Thus, the governing equations are:

$$
\begin{aligned}
\boldsymbol{\varepsilon}(t) & =\frac{1}{2}\left(\boldsymbol{\nabla u}(t)+(\boldsymbol{\nabla u}(t))^{T}\right) & & \text { in } \Omega \\
\boldsymbol{\sigma}(t) & =\mathcal{A}: \boldsymbol{\varepsilon}(t) & & \text { in } \Omega \\
\boldsymbol{\nabla} \cdot \boldsymbol{\sigma}(t) & =\mathbf{0} & & \text { in } \Omega \\
\boldsymbol{u}(t) & =\boldsymbol{u}_{d}(t) & & \text { on } \partial_{u} \Omega \\
\boldsymbol{\sigma}(t) \cdot \boldsymbol{n} & =\boldsymbol{F}_{d}(t) & & \text { on } \partial_{F} \Omega \\
\boldsymbol{\sigma}(t) \cdot \boldsymbol{n}_{\Gamma} & =\boldsymbol{f}(\llbracket \boldsymbol{u}(t) \rrbracket, \lambda) & & \text { on } \Gamma_{t}
\end{aligned}
$$

where $\mathcal{A}$ denotes the elasticity tensor, $\boldsymbol{n}$ the outward unit normal to any point of the boundary $\partial \Omega, \boldsymbol{n}_{\Gamma}$ the unit normal to any point on the crack surface $\Gamma$ and $f$ the cohesive law relationship where $\lambda$ is an internal variable that describes the damage of the interface.

In order to preserve an accurate numerical solution, a hybrid cohesive formulation is chosen. Like in [13], the initial formulation of the cohesive interface is based on a discontinuous Galerkin (DG) method. When a specific stress threshold is reached on any point of the interface, a classical linear dissipative cohesive model is applied. This approach leads to the following variational formulation for the problem at hand: find $\boldsymbol{u}$ that verifies $\forall \boldsymbol{v} \in\left\{\boldsymbol{w} \in H^{1}(\Omega), \boldsymbol{w}=\mathbf{0}\right.$ on $\left.\partial_{U} \Omega\right\}$ and $t \in\left[0, t_{f}\right]$ :

$$
\begin{aligned}
& \int_{\Omega} \boldsymbol{\varepsilon}(t): \mathcal{A}: \boldsymbol{\nabla} \boldsymbol{v} \mathrm{d} \Omega-\int_{\Gamma_{t}}(1-\alpha)\left(\langle\mathcal{A}: \boldsymbol{\nabla} \boldsymbol{v}\rangle:\left(\boldsymbol{n}_{\Gamma} \otimes \llbracket \boldsymbol{u}(t) \rrbracket\right)\right. \\
&\left.-\langle\boldsymbol{\sigma}(t)\rangle:\left(\boldsymbol{n}_{\Gamma} \otimes \llbracket \boldsymbol{v} \rrbracket\right)+\kappa\left(\llbracket \boldsymbol{u}(t) \rrbracket \otimes \boldsymbol{n}_{\Gamma}\right): \mathcal{A}:\left(\boldsymbol{n}_{\Gamma} \otimes \llbracket \boldsymbol{v} \rrbracket\right)\right) \mathrm{d} S \\
& \quad=\int_{\partial_{F} \Omega} \boldsymbol{F}_{d}(t) \cdot \boldsymbol{v} \mathrm{d} S+\int_{\Gamma(t)} \alpha \boldsymbol{f}(\llbracket \boldsymbol{u}(t) \rrbracket, \lambda) \cdot \llbracket \boldsymbol{v} \rrbracket \mathrm{d} S
\end{aligned}
$$

where, for any point of the surface $\Gamma_{t}$ and any generic fields $q_{1}, q_{2}$ defined on the lower and upper crack faces $\Gamma_{t, 1}$ and $\Gamma_{t, 2}$, one has set:

$$
\langle q\rangle:=\frac{1}{2}\left(q_{1}+q_{2}\right), \quad \llbracket \boldsymbol{q} \rrbracket:=\boldsymbol{q}_{2}-\boldsymbol{q}_{1},
$$

with $\boldsymbol{n}_{\Gamma}$ is the unit normal on $\Gamma_{t}$ pointing from $\Gamma_{t, 1}$ to $\Gamma_{t, 2}$.

The scalar $\alpha$ is initially set to 0 for $t=0$. The criterion on the stress is defined such that for any point of the surface $\Gamma_{t}, \alpha$ is set to 1 once $\langle\boldsymbol{\sigma}(t)\rangle:\left(\boldsymbol{n}_{\Gamma} \otimes \boldsymbol{n}_{\Gamma}\right)>\sigma_{F}$. To take into account the damage accumulated on the surface, this process is irreversible.

If $\alpha=0$, the applied formulation is based on the Nitsche internal penalty method [16], where $\kappa$ is a penalty coefficient: if $\kappa$ is chosen equal to $C / h$ in the discrete solution process with $C$ positive and large enough and $h$ the smallest surface element characteristic size, the stability and convergence are guaranteed. 
If $\alpha=1$, a simple cohesive model (mostly built for mode I loading) is activated. First, an interface damage parameter $\lambda$ is used, defined, only if $a=1$, by:

$$
\lambda(0)=0 \quad \text { and } \quad \lambda(t)=\min \left(1, \max _{\tau \in[0, t] \mathrm{AND} \llbracket \boldsymbol{u} \rrbracket \cdot \boldsymbol{n}_{\Gamma}>0} \frac{\|\llbracket \boldsymbol{u}(t) \rrbracket\|}{u_{F}}\right),
$$

so that the interface behaviour can be defined as (Figure 1):

- if $\llbracket \boldsymbol{u} \rrbracket \cdot \boldsymbol{n}_{\Gamma}>0$, a traction loading is applied:

$$
\boldsymbol{f}(\llbracket \boldsymbol{u}(t) \rrbracket, \lambda)= \begin{cases}\frac{\llbracket \boldsymbol{u}(t) \rrbracket}{\lambda u_{F}}(1-\lambda) \sigma_{F}, & \text { if } \lambda<1 \\ 0, & \text { otherwise }\end{cases}
$$

- otherwise, a compressive loading is applied:

$$
\boldsymbol{f}(\llbracket \boldsymbol{u}(t) \rrbracket, \lambda)=K_{\mathrm{comp}} \llbracket \boldsymbol{u}(t) \rrbracket
$$

where $u_{F}$ denotes the critical jump at the interface where damage is triggered, $\sigma_{F}$ is computed related to the critical energy release rate of the material, and $K_{\text {comp }}$ is a penalty coefficient to apply perfect contact when a compression loading is applied close to the considered $\Gamma$ surface point.

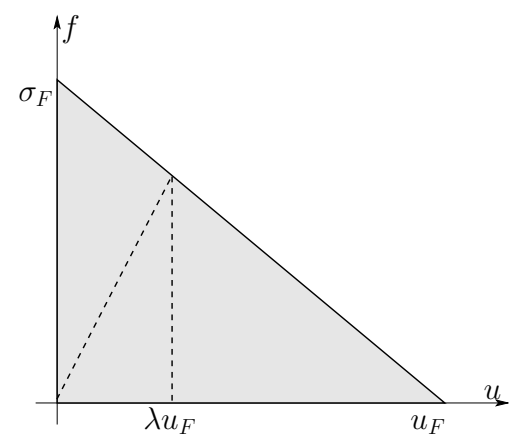

Figure 1: Cohesive law adopted for the crack propagation when the threshold has been reached.

\section{Adaptive refinement approach for cohesive zone model based on a remeshing process}

Using a discretized version of the formulation given in the last section and considering a quasi-static loading that ensures a stable evolution of the system, it is possible to solve the crack growth problem on a completely predefined cohesive zone, using a standard NewtonRaphson algorithm. The "process zone" is defined as the group of cohesive elements which contains, at the current time $t$, at least one integration point that satisfies $0<\lambda<1$.

To solve such problems accurately usually requires a very fine mesh in the "process zone". Thus, if the same mesh is used to model the problem over the complete evolution 
duration $\left[0, t_{f}\right]$, mesh refinement is needed on the whole crack path, making the overall solution process computationally expensive. In this section, an efficient adaptive remeshing approach is presented, which considerably accelerates the numerical solution of such problems.

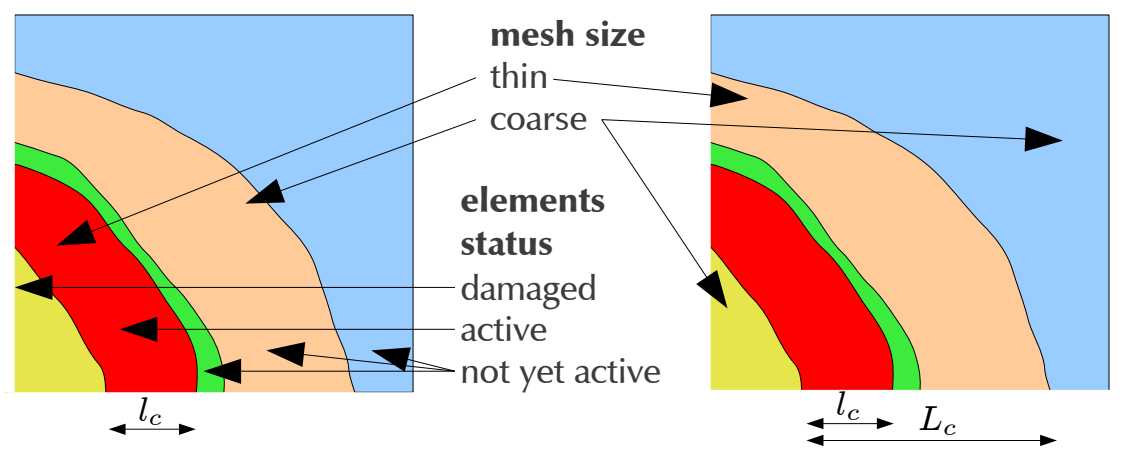

Figure 2: Representation of the cohesive surface, the relationship between the mesh size and the interface elements' damage status during a remeshing step.

During the adaptive refinement process, a prescribed minimal element size $h$ is set based on considerations related to the dissipative model and the size of the "process zone": the characteristic length $\ell_{c}$ of the "process zone" is defined as the smallest distance between two material points whose state evolves from $\lambda=0$ to $\lambda=1$. The usual requirement is that the cohesive elements in this zone must have a maximal size of $h=$ $\ell_{c} / 4$. Then, a zone built from the non-damaged elements within a distance $L_{c}$ (a second characteristic length) to the actual "process zone" is defined. In this region, the prescribed mesh size is set to $h=\ell_{c}$. Elsewhere on the surface and in the volume mesh, the remeshing algorithm is free to apply the most suitable element size.

During the computation, the "process zone" evolves (Figure 2). If it reaches a "coarse" cohesive element, the solution state is reverted to the last converged state, where all active cohesive elements size is prescribed (in the "refined" zone). A remeshing process is then triggered in order to generate a more suitable mesh from the current converged solution. A mesh refinement is imposed in an updated $L_{c}$-based zone, coarsening is applied where the cohesive elements are broken, and the exact topology of the elements in the "process zone" is preserved.

Before computing the next problem increment, a field transfer process is required. For both the bulk linearly-elastic behaviour and the non-linearities concentrated on the cohesive surfaces:

- The integration point transfer is only crucial for the "process zone" elements; elsewhere, only binary values of $\lambda$ must be correctly transferred because of the linear behaviour. Thus, a "closest integration point" transfer technique is used, separating volumetric elements and the cohesive ones. Since the topology is exactly preserved on the "process zone", the static equilibrium is perfectly kept for these elements. 
- Since there is a localized discontinuity when the displacement field crosses damaged cohesive elements, the transfer of nodal unknowns is complex. However, for the sake of simplicity, since the problem is set in a linear elastic constitutive framework, the displacement fields are initialized to zero everywhere.

An equilibrium increment is then performed in order to revert the problem back to its state before the field transfers by applying the complete loading from $t=0$ to the current time within one step. The global evolution on this interval has to be almost completely linear because of the specific behaviour models of the bulk material and the cohesive interfaces.

\section{Crack direction prediction and remeshing process}

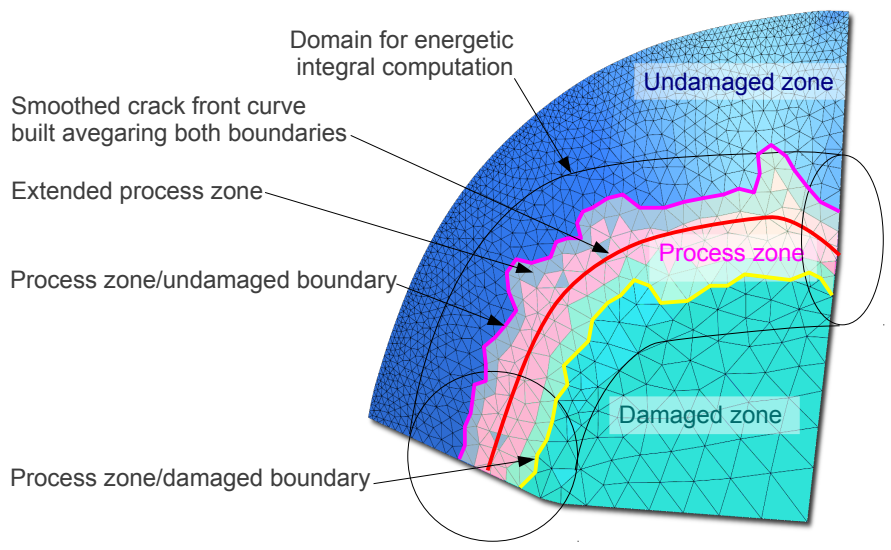

Figure 3: Representation of an approximated crack front and an integration volume for stress intensity factor computations.

In this section, a specific crack direction prediction criterion, used for updating the crack path during the simulation, is discussed. To process complex crack path updates while using cohesive zone models, a global energetic approach is chosen. Since a linear elastic behaviour is used for the bulk material, if the ratio between the crack surface area and the surface of the "process zone" is large enough, the considered problem can be treated as a small-scale yielding fracture problem. Thus, a direction prediction criterion formulated for linear elastic fracture mechanics can be used.

In order to apply such criteria to general 3D problems with cracked surfaces, the first step consists of building a crack front (Figure 3):

1. Discrete cohesive zone solutions usually produce a "process zone" made of noncontiguous elements, which make it particularly difficult to identify an approximated crack front. In order to limit these artefacts and build a more regular surface, the "process zone" is extended to all the cohesive elements that have a least one node linked to it. 

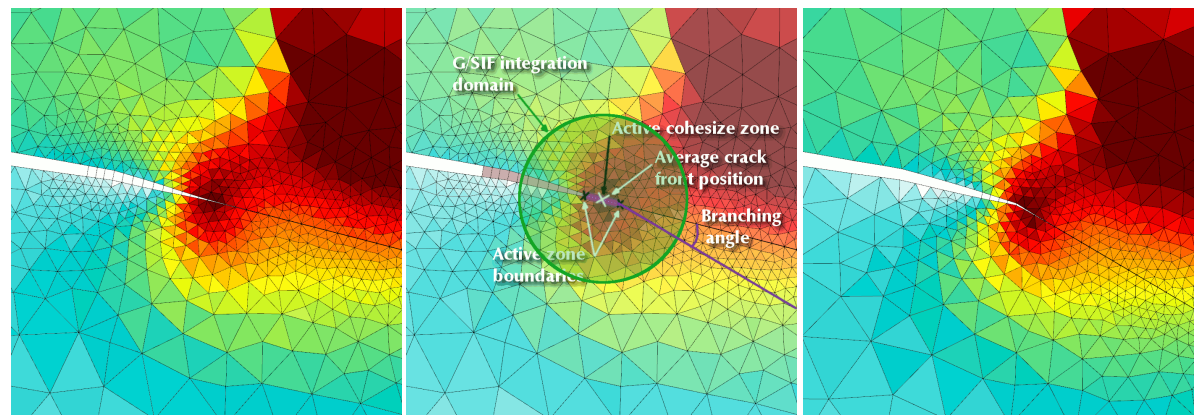

Figure 4: Remeshing process with cohesive surface reorientation.

2. Segments on the boundary between the extended zone and the completely damaged elements (where the damage internal variable $\lambda=1$ ) are ordered and linked together to build a continuous polygon. Using a smoothing technique [6], an average curve $\gamma_{D}=\left\{\gamma_{D}(s), s \in S\right\}$ is built (where $s$ denotes an arc-length coordinate).

3. By using the same technique, an average curve $\gamma_{U}$ is built on the interface between the undamaged elements.

4. Finally, the averaged crack front $\gamma$ is built according to:

$$
\gamma(s)=\frac{1}{2}\left(\gamma_{U}(s)+\gamma_{D}\left(s_{m}\right)\right) \text { where } s_{m}=\operatorname{argmin}_{\xi}\left\|\gamma_{U}(\mathrm{~s})-\gamma_{\mathrm{D}}(\xi)\right\|
$$

In other words, $\gamma(s)$ is the midpoint between a given point $\gamma_{U}(s)$ and the point of $\gamma_{D}$ closest to the latter.

To define the new crack orientation, an interaction integral computation [11] is carried out on the crack front [6]. Usually the volume mesh diameter for the energetic integral computation is about ten times that of the "process zone". A maximal opening stress criterion is then used: if the extracted stress intensity factor value in mode II $K_{I I}$ is sufficiently high compared to mode I value $K_{I}$, the branching angle $\beta$ is obtained in order to satisfy:

$$
K_{I} \sin (\beta)+K_{I I}(3 \cos (\beta)-1)=0
$$

If the criterion indicates that the crack direction changes, an adaptive remeshing process is used [7] (with respect to the considerations expressed in the last section, see figure 4):

(i) a surface mesh is generated as an extension of length $L_{c}$ of the undamaged "process zone" boundary $\gamma_{U}$ in the directions given by the criterion (it should therefore not intersect the actual "process zone").

(ii) the current mesh is cut by the previously generated surface.

(iii) the previously undamaged elements are deleted, and the new crack surface is generated as a union of the previous zone linked to the "process zone" and the new generated extension. 
(iv) an adaptive remeshing stage is applied, where a prescribed element size $\ell_{c}$ is set for the newly generated undamaged cohesive elements. The "process zone" topology is preserved whereas the totally damaged elements are removed.

(v) the transfer technique presented in the last section is used once again.

If no branching occurs, the computation continues until the "process zone" reaches the end of the predefined cohesive surface. At this point, another remeshing process is triggered at the beginning of the current time increment and the surface is extended by a length $L_{c}$.

\section{$5 \quad$ Numerical assessments}

In this last section, two applications of the developed algorithm are presented. The first one is focused on the adaptive remeshing technique, which can considerably reduce the computational time in case of cohesive zone model with a predefined crack path. The second one is based on the simulation of a complex, stable crack propagation in a bimaterial specimen, using the adaptive crack path prediction technique.

\subsection{Example 1: adaptative remeshing for a double cantilever beam}

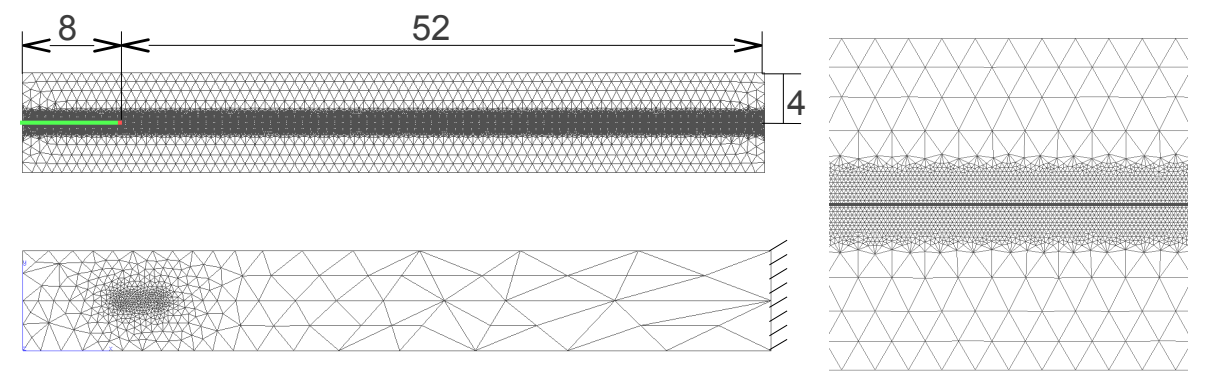

Figure 5: Example 1: fixed and initially adapted meshes for DCB propagation.

In this section, a simple 2D double cantilever beam (DCB) under monotonous traction is considered. The material is linear elastic (Young modulus: $200000 \mathrm{MPa}$, Poisson ratio: 0.3 ). Details about the geometry are given on figure 5 , where all units are millimetres. A notch has been inserted on the mid-plane of the beam with an initial length of $8 \mathrm{~mm}$. A zero displacement is prescribed to the right end of the specimen, whereas vertical increasing displacements are applied to each left corner along opposite directions so as to open the notch. The cohesive zone model is based on the hybrid DG/linear dissipative model presented in section 2 with $\sigma_{F}=100 \mathrm{MPa}$ and $u_{F}=1.10^{-3} \mathrm{~mm}$.

First a numerical simulation is carried out using a single mesh i.e. without adaptive remeshing. A fine discretisation is imposed near the dissipative interfaces (Figure 5). Both cohesive elements and bulk material elements feature quadratic shape functions for a total of 173460 DOFs. 


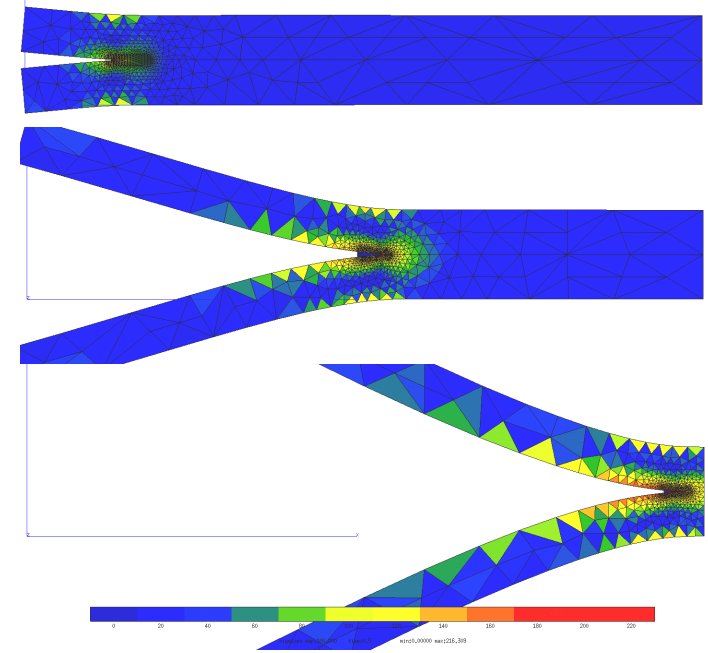

Figure 6: Example 1: evolution of adapted mesh during propagation (contour map of von Mises equivalent stress).

Next, an initial adaptive mesh is set up using the considerations exposed in section 3. The size of the elements in the refined zone is the same as in the previous, fixedmesh, simulation. This second mesh initially involves 6273 DOFs (and stays under 7000 DOFs during the whole crack propagation, see figure 6, while undergoing 221 successive remeshing steps). Thus, the overall computational time for 400 loading increments (using an updated Newton-Raphson procedure) is less than 15 minutes, instead of over 3 hours using the non-adaptive fine mesh.

\subsection{Example 2: crack propagation with a complex crack path}

This example aims at assessing and demonstrating the efficiency and robustness of the method. In this respect, a critical crack growth problem is simulated for a modified CT specimen that is specially designed to ensure that the crack growth is stable. The 3D specimen geometry (Figure 7 , right) is obtained from the 2D geometry in the $(x, y)$-plane of Figure 7 (left) by (i) extending it along the $z$ (out-of-plane) coordinate so as to create a $0.1 \mathrm{~mm}$ uniform thickness (with the $z=0$ plane as symmetry plane), and (ii) subsequently applying the geometrical transformation

$$
\boldsymbol{x} \mapsto \boldsymbol{x}+.005 y|y| \boldsymbol{e}_{z} \quad \text { (lengths in millimetres) }
$$

The specimen thickness thus varies between $0.1 \mathrm{~mm}$ (in the central part $y=0$ ) and $9.1 \mathrm{~mm}$ (at the top or bottom right edges). The mechanical loading is defined by:

- zero displacements prescribed on the right side;

- increasing vertical displacements applied at the upper left and the lower right corners. 

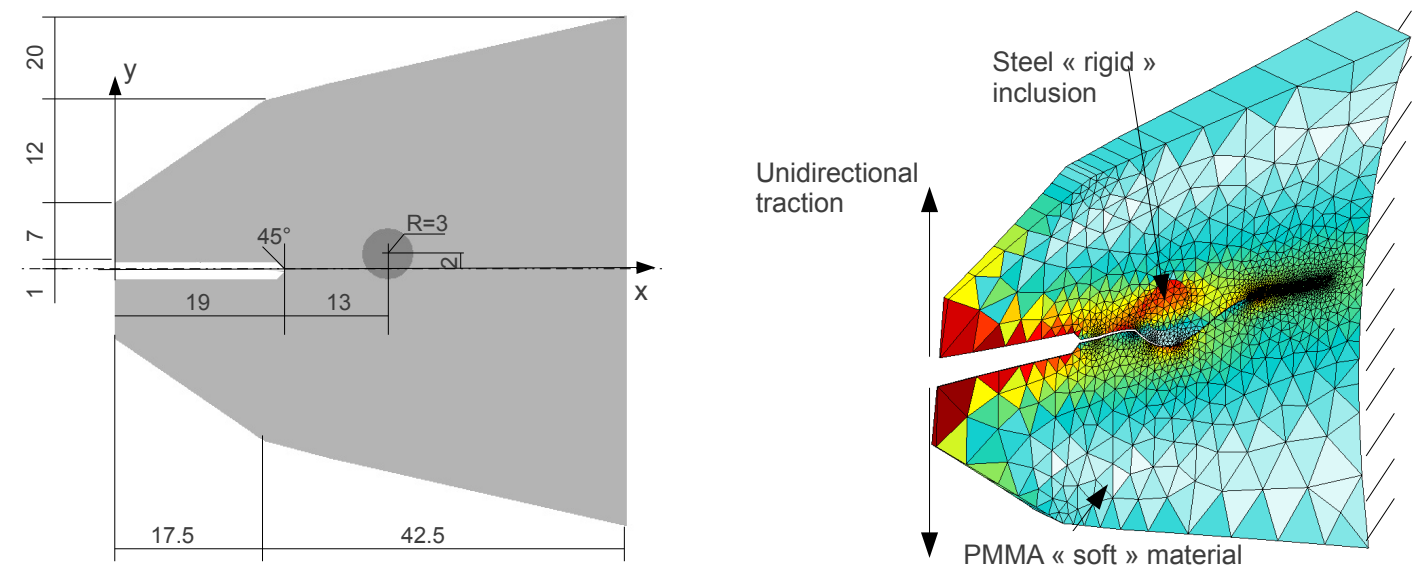

Figure 7: Example 2: specimen geometry (units are millimetres) and loading conditions.

The structure is mostly made of PMMA (Young modulus: $2380 \mathrm{MPa}$, Poisson ratio: 0.35), with a rigid steel inclusion (Young modulus: $200000 \mathrm{MPa}$, Poisson ratio: 0.3) inserted in the central region of the specimen. The model assumes perfect bonding conditions between the two materials, which are both considered linear elastic. The proposed cohesive model is used on the dissipative interface with $\sigma_{F}=100 \mathrm{MPa}$ and $u_{F}=1 \cdot 10^{-3} \mathrm{~mm}$. The numerical study will focus on the crack path prediction, the whole crack evolution being stable by design of the specimen.

The crack path evolution predicted using the present adaptive remeshing method is shown on figure 8. In this case, 62 remeshing steps have been performed. A usual linear elastic crack propagation simulation has also been performed for comparison (with the same branching criterion, prescribed advance step and linear loading conditions). The two resulting predicted crack paths are shown on figure 9; they are quite similar, although they do differ in the region where the cracks avoids the steel inclusion.

\section{Conclusion}

In this paper an adaptive remeshing strategy has been presented, aiming at considerably reducing computational costs for predefined crack path and update the cohesive interface geometry by using an energetic linear elastic criterion. The preliminary numerical examples presented herein demonstrate the efficiency of this approach for quasi-2D problems. Future extensions will be focused on the simulation of real 3D configurations and the extension of the approach to more complex bulk material behaviours, which add challenges such as maintaining the static equilibrium during the field transfer process.

\section{References}

[1] Alfano, G., Crisfield, M. A. Finite element interface models for the delamination analysis of laminated composites: mechanical and computational issues. Int. J. Num. Meth. Eng., 50:1701-1736 (2001). 

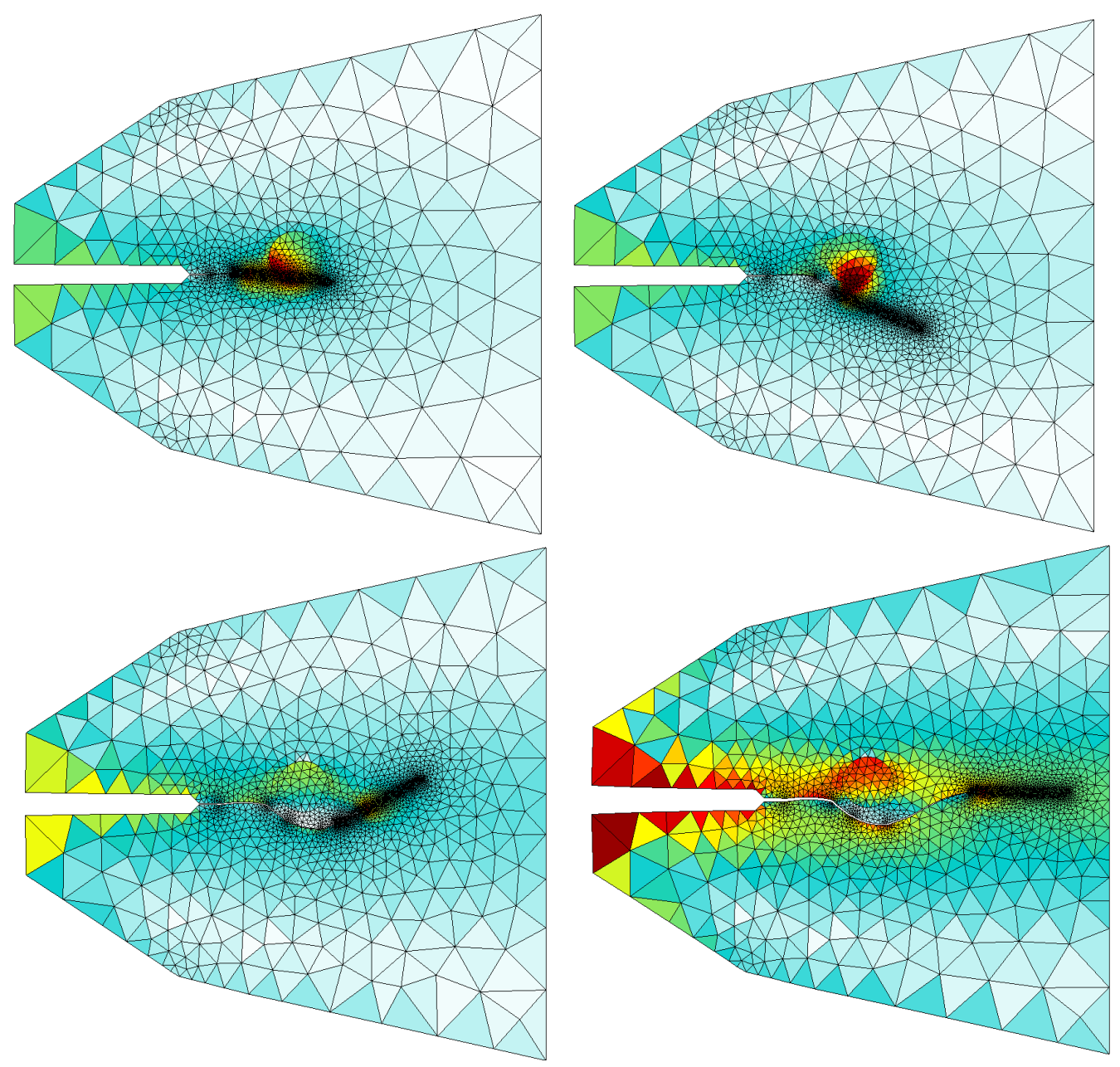

Figure 8: Evolution of crack path during the problem evolution using the adaptive cohesive zone modelling (von Mises isovalues).

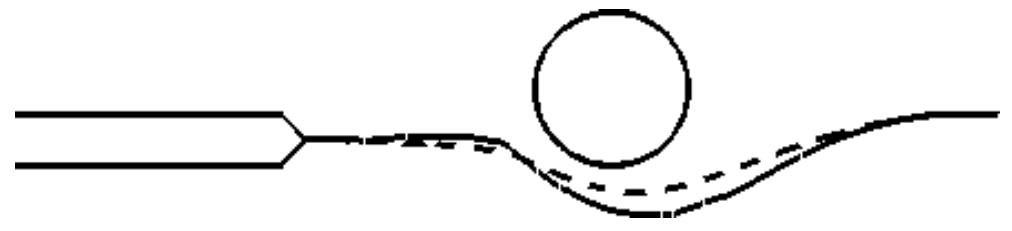

Figure 9: Comparison of crack paths obtained via adaptive cohesive model (plain line) and linear elastic fracture mechanics (dashed).

[2] Barenblatt, G.I. The mathematical theory of equilibrium cracks in brittle failure. Adv. Appl. Mech., 7:55-129 (1962).

[3] Bechet, E., Minnebo, H., Moes, N., Burgardt, B. Improved implementation and robustness study of the X-FEM for stress analysis around cracks. Int. J. Num. Meth. Eng., 64:1033-1056 (2005).

[4] Bonnet, M. Stability of crack fronts under Griffith criterion: a computational ap- 
proach using integral equations and domain derivatives of potential energy. Comput. Meth. Appl. Mech. Eng., 173:337-364 (1999).

[5] Chessa, J., Smolinski, P., Belytschko, T. The extended finite element method (XFEM) for solidification problems. Int. J. Num. Meth. Eng., 53:1959-1977 (2002).

[6] Chiaruttini, V., Feld-Payet, S., Rannou, J. Some recent advances in remeshing techniques for complex 3D crack growth simulation. ODAS meeting, DLR, Braunschweig, Germany (2012).

[7] Chiaruttini, V., Feyel, F., Chaboche, J. L. A robust meshing algorithm for complex 3D crack growth simulation. European Conference on Computational Mechanics, Paris, France (2010).

[8] Destuynder, P., Lescure, S., Djaoua, M. Some remarks on elastic fracture mechanics. Journal de Mécanique Théorique et Appliquée, 2:113-135 (1983).

[9] Dugdale, D.S. Yielding of steel sheets containing slits. J. Mech. Phys. Solids, 8:100104 (1960).

[10] Geißler, G., Kaliske, M. Discrete crack path prediction by an adaptive cohesive crack model. Eng. Fract. Mech., 77:3541-3557 (2010).

[11] Gosz, M., Dolbow, J., Moran, B. Domain integral formulation for stress intensity factor computation along curved three-dimensional interface cracks. Int. J. Solids Struct., 35:1763 - 1783 (1998).

[12] Levy, S., Seagraves, A., Molinari, J-F., Radovitzky, R. Discontinuous Galerkin method applied to fragmentation of heterogeneous materials. 9e Colloque en calcul des structures, Giens, France (2009).

[13] Mergheim, J., Kuhl, E., Steinmann, P. A hybrid discontinuous Galerkin/interface method for the computational modelling of failure. Commun. Numer. Meth. Engng, 20:511-519 (2004).

[14] Mi, Y., Crisfield, M. A., Davies, G. A. O., Hellweg, H. B. Progressive delamination using interface elements. J. Compos. Mater., 32:1246-1272 (1998).

[15] Moes, N., Dolbow, J., Belytschko, T. A finite element method method for crack growth without remeshing. Int. J. Num. Meth. Eng., 46:131-150 (1999).

[16] Nitsche, J. A. Uber ein Variationsprinzip zur Losung Dirichlet-Problemen bei Verwendung von Teilrau- men. In Abh. Math. Sem. Univ. Hamburg, pp. 9-15 (1971).

[17] Zi, G., Belytschko, T. New crack-tip elements for XFEM and applications to cohesive cracks. Int. J. Num. Meth. Eng., 57:2221-2240 (2003). 